\title{
EM TORNO DO RENDIMENTO ESCOLAR: ADAPTAÇÃO DE UM INVENTÁRIO DE ESTRATÉGIAS DE ESTUDO PARA UMA POPULAÇÃO ESTUDANTIL PORTUGUESA*
}

\author{
Célia Ribeiro**
}

\section{INTRODUÇÃO}

A suposição de que o rendimento escolar pode situar-se muito abaixo das potencialidades dos alunos tem vindo a tomar corpo (v.g., Benet et al., 1990). De facto, considerando diferentes níveis de ensino, inclusive o universitário, não parece ser possível explicar os desempenhos na aprendizagem tendo apenas em conta factores de ordem intelectual. A necessidade de investigar outras variáveis, tanto cognitivas como motivacionais, para esclarecer as diferenças de realização entre os alunos é, hoje em dia, amplamente aceite (Fontaine, 1990).

Esta ideia adquire tanta ou mais importância se pensarmos que o aluno, nomeadamente em níveis de ensino mais elevados, surge pouco apoiado perante o insucesso, pouco motivado e mesmo incapaz de dar resposta à grande diversidade de situações escolares com as quais se confronta. $O$ consumo de informação em catadupa, fornecida em enormes quantidades, de um modo por vezes não estruturado e a um ritmo bastante intenso, dificulta a sua assimilação, já que raramente é ensinado ao aluno como processar essa informação, de modo a organizá-la, estruturá-la, seleccioná-la, generalizando-a e transmitindo-a (Carvalho et al., 1987).

Realmente, tem-se verificado que a maioria dos alunos de nível universitário tende a usar um número limitado de estratégias para aprender e, quando sabe da

** Instituto Universitário de Desenvolvimento e Promoção Social - Pólo de Viseu da Universidade Católica Portuguesa. 
sua existência, não tem consciência de como e quando as usar (Simpson et al., 1994). Refira-se, também, que em muitas disciplinas e cursos em geral, os alunos não são explicitamente informados sobre o tipo específico de performance que lhes será solicitado no processo de avaliação, dificultando a selecção de estratégia(s) adequada(s) para o estudo daquele material específico.

Parece, então, que ajudar os alunos a desenvolver modos eficazes para lidar com a informação proveniente do meio e com os seus próprios processos de pensamento deve ser um dos objectivos do sistema educativo actual. De facto, diversas investigações têm encontrado uma relação entre estratégias de estudo e realização escolar (v.g., Camp, 1990; Elshout \& Veenman, 1992), em que a utilização de determinadas estratégias, tais como a elaboração e organização, constitui um envolvimento cognitivo activo, que resulta em níveis elevados de rendimento escolar (Pintrich \& De Groot, 1990). Constituindo-se como ferramentas cognitivas, nas palavras de Paris \& Winograd (1990), a utilização de estratégias, quando consciente e adequada, pode melhorar, significativamente, o nível de realização e, como tal, o sucesso escolar.

Apesar desta questão já ter sido objecto de diversas investigações em outros países (v.g., EUA: Biggs, 1984; Dansereau, 1978, 1985, 1988; Weinstein, 1988; Espanha: Moreno \& Di Vesta, 1991), em Portugal, poucos estudos têm sido levados a cabo (v.g., Silva \& Sá, 1989), nomeadamente a nível do ensino superior.

Assim, na tentativa de dar um contributo para o esclarecimento da complexidade inerente ao fenómeno educativo, a presente investigação tem como objectivos, por um lado, a adaptação de um instrumento de avaliação de estratégias de estudo em estudantes universitários: o Cognitive Skills Inventory e, por outro, a verificação da relação entre as estratégias de estudo (de integração, de repetição, de monitorização e de coping) e o rendimento e a história escolar.

\section{ALGUMAS CONSIDERAÇÕES EM TORNO DO CONCEITO ESTRATÉGIA}

A crescente atenção e importância dada às teorias que procuram explicar os processos subjacentes à aprendizagem influenciou radicalmente as concepções e intervenções na área das estratégias de estudo. Enquanto a perspectiva behaviorista as encara como um conjunto de comportamentos separados e independentes, interessando apenas conhecer os componentes da actividade de estudo para se poder promover a aquisição dos comportamentos que a constituem, a perspectiva cognitivista, mais especificamente os modelos de processamento da informação, forneceu dados sobre os sistemas de atenção e memória, que vieram influenciar e fundamentar grande parte dos programas de intervenção na área das estratégias ${ }^{1}$. 
Apesar do estudo sistemático das estratégias só se ter iniciado nos anos 60/70, a sua importância data de muito antes. Por exemplo, já nos tempos de Aristóteles, a imagem assumia um papel central na retenção da informação, e a associação de ideias um papel central na sua recordação. $\mathrm{Na}$ época dos professores de retórica, tais como Cícero e Quintiliano, verifica-se, também, o interesse pela criação de relações entre a experiência e a nova informação, como uma técnica pedagógica eficaz, através da criação de imagens interactivas entre as ideias antigas e os novos eventos. Uma grande parte da retórica consistiu na aprendizagem do modo mais adequado de memorizar as sequências nos discursos, pois o papel e o lápis não estavam prontamente disponíveis para tirar notas ou fazer esboços. Em vez disso, os oradores utilizavam mnemónicas de imagens, derivadas do modelo de memória de Aristóteles, que enfatizou a colocação de pontos numa sequência linear, em que cada ponto representava uma imagem que envolvia a interacção com um objecto familiar facilmente recordável (Cf. Wittrock, 1988).

Estes interesses também se ajustam aos achados iniciais, mas esquecidos, de começos do século XX, pois as sementes para o estudo das estratégias já se encontravam presentes nos trabalhos desenvolvidos por Binet ${ }^{2}$, Baldwin, Piaget, Dewey, Thorndike e outros, mas que, no entanto, levaram muito tempo a germinar ${ }^{3}$.

\subsection{Tentativa de definição}

$\mathrm{Na}$ literatura, embora se verifique um consenso entre os diversos autores acerca da importância e utilidade das estratégias para uma adequada realização escolar, nota-se ainda uma lacuna em termos de uma definição unívoca e precisa. Observa-se uma grande diversidade em torno deste conceito, encontrando-se algumas definições de âmbito mais geral, outras mais específicas, para além de algumas confusões em termos de terminologias e grande complexidade em termos de taxonomias ${ }^{4}$.

Enquanto alguns autores (v.g., Mayer, 1988) entendem as estratégias como comportamentos levados a cabo pelo aprendiz com o objectivo de influenciar o modo de processamento da informação (por exemplo, sublinhar as ideias-chave num texto, ou parafrasear uma nova informação), outros (v.g., Brooks et al., 1985; Levin \& Pressley, 1985) consideram que são procedimentos direccionados para um objectivo, são planeadas ou intencionalmente evocadas antes, durante e após a realização da tarefa, isto é, auxiliam a execução, a regulação e a avaliação desta.

Paris et al. (1983) designam-nas de «skills under consideration», uma vez que requerem recursos atencionais e podem ser analisadas, relatadas e modificadas. Os mesmos autores referem ainda que, para uma actividade ser considerada uma 
estratégia, deve ser seleccionada pelo aprendiz de actividades alternativas e ter como objectivo atingir uma meta ou completar uma tarefa.

Deshler \& Lenz (1989, in Lens, 1992) definem-nas como o modo de abordar uma tarefa, incluindo o modo como se pensa e age quando se planeia, executa e avalia o desenvolvimento e os resultados numa tarefa. Realçam, pois, para além da componente comportamental (o que o sujeito actualmente faz), a componente cognitiva (o que se passa no pensamento do sujeito) que, conjuntamente, orientam o desenvolvimento e a avaliação da tarefa.

Silva \& Sá (1993) salientam ainda que, a um nível mais complexo, as estratégias podem ser definidas como planos formulados pelos alunos para atingirem objectivos de aprendizagem (por exemplo, realizar um conjunto de acções para a elaboração de uma composição escrita) e, a um nível mais específico, como qualquer procedimento adoptado para a realização de uma determinada tarefa (por exemplo, rever para corrigir os erros ortográficos). Deste modo, as estratégias podem abranger diversos tipos de aplicações: umas são mais específicas à tarefa (por exemplo, sublinhar um texto), outras são mais gerais e podem ser adoptadas em tarefas de características muito diferentes (planear: um trabalho escrito, a leitura de um texto ou a resolução de um problema).

De referir, ainda, que a utilização de uma determinada estratégia pode ter como objectivo afectar o modo como se selecciona, adquire, organiza ou integra o novo conhecimento, bem como manter um estado motivacional e afectivo adequado para a realização da tarefa, estratégias estas designadas por Dansereau (v.g., 1978, 1985) de primárias e de suporte, respectivamente 5 .

Em síntese, podemos verificar, segundo as definições apresentadas e outras encontradas na literatura, que as estratégias podem ser entendidas como comportamentos/procedimentos levados a cabo pelo aprendiz com o objectivo de influenciar o modo como processa a informação, através da activação, controlo e regulação dos processos cognitivos. São conscientemente planeadas ou intencionalmente evocadas antes, durante e após a realização da tarefa. Observa-se,-assim, a importância atribuída à finalidade, à natureza intencional do comportamento como uma característica crucial daquilo que podemos denominar estratégia.

\section{METODOLOGIA}

\subsection{Amostra}

Constituída, no total, por 588 estudantes universitários, sendo que:

No Estudo 1 participaram 52 estudantes, de ambos os sexos (32 do sexo feminino e 20 do sexo masculino), com idades compreendidas entre os 20 e os 25 anos, 
do $3^{\circ}$ ano do Curso de Licenciatura em Gestão e Desenvolvimento Social, da Universidade Católica Portuguesa, Pólo de Viseu.

Nos Estudos 2 e 3 participaram 536 estudantes, de ambos os sexos (390 do sexo feminino e 146 do sexo masculino), com idades compreendidas entre os $20 \mathrm{e}$ os 30 anos, que frequentavam o $3 .^{\circ}$ e o $4 .^{\circ}$ anos do Ramo de Formação Educacional da Faculdade de Letras da Universidade de Coimbra ${ }^{6}$.

\subsection{Instrumento}

O Cognitive Skills Inventory de Francis Di Vesta (1986), o qual designamos por Inventário de Estratégias de Estudo (IEE), foi elaborado com base na suposição de que a motivação e as atitudes são processos (entendidos, não de maneira isolada, mas interligados funcionalmente entre si) que influenciam a capacidade dos estudantes para enfrentar as distracções e a ansiedade (Moreno \& Di Vesta, 1991).

De acordo com Moreno \& Di Vesta (1991) é suposto que a motivação e as atitudes estejam relacionadas com o controlo do sistema de processamento de informação. Ou seja, que permitam: prestar atenção, o que possibilita a recepção de informação através do registo sensorial; dirigir especificamente a atenção, o que possibilita limitar a passagem da informação relevante do registo sensorial para a memória a curto prazo; a utilização da repetição, o que possibilita a manutenção da informação na memória a curto prazo; a integração da nova informação no conhecimento anteriormente adquirido, o que possibilita tornar a aprendizagem significativa e a actividade de monitorização, que auxilia a planificação do estudo, o que possibilita a verificação dos objectivos propostos e a proceder a alterações nas estratégias de estudo, no caso de os objectivos não estarem a ser atingidos.

O IEE, na versão que utilizámos, é constituído por 40 itens (reportando-se, uns, a estratégias de selecção, de organização e de codificação da informação e, outros, a estratégias de metacognição ${ }^{7}$ ), distribuídos por quatro factores ou subescalas complementares: Integração, Repetição, Monitorização e Coping.

O factor Integração refere-se ao processamento profundo de informação ${ }^{8}$ o estudante, ao elaborar, ao sumariar e ao parafrasear as ideias contidas num documento de estudo, organiza-as e relaciona-as com as ideias anteriores. Esta estratégia permite o estabelecimento de uma rede significativa de conhecimentos na memória a longo prazo. E, neste sentido, compensa as limitações da memória a curto prazo.

O factor Repetição refere-se ao processamento superficial de informação: o estudante organiza, ordena e repete as ideias no sentido de as reter durante algum tempo na memória a curto prazo. 
O factor Monitorização pressupõe que as metacognições no estudo são relevantes para a sua eficácia. Os itens que o constituem reportam-se à tomada de consciência da necessidade de determinação de objectivos e da verificação do estudo, bem como do recurso a estratégias de «reparação» quando são detectadas falhas na compreensão.

O factor Coping ${ }^{9}$ surge com base na ideia de que o lutar com a distracção se encontra relacionado com a eficácia do estudo. Os itens que o constituem reportam-se, de maneira estrita, à (in)capacidade para lidar com situações geradoras de ansiedade e englobam actividades que requerem um empenhamento activo para reduzir a fadiga, a frustração e o stress, que as situações de estudo por vezes envolvem.

Uma pontuação elevada nesta subescala indica dificuldades ou, mesmo, incapacidade para lutar com situações ansiógenas, como, por exemplo, a preparação e a realização de um exame.

No que concerne ao modo de preenchimento do $I E E$, os sujeitos são solicitados a explicitar a frequência de utilização de determinadas actividades de estudo, através de uma escala de tipo Likert de 5 pontos ( $\mathbf{A}=$ nunca faço isto a $\mathbf{E}=$ sempre faço isto).

\subsection{Procedimento}

A investigação foi empreendida em três fases consecutivas:

\section{Estudo 1: Primeira fase de adaptação}

Consistiu na tradução do Cognitive Skills Inventory, em que procurámos respeitar as intenções originais do seu autor. A este trabalho seguiu-se a administração do Inventário, já traduzido, à Amostra 1.

Foi, também, pedido aos sujeitos que respondessem a um Questionário de Avaliação de Instrumentos.

\section{Estudo 2: Pré-teste}

Este estudo teve como objectivo, por um lado, obter uma configuração factorial do $I E E$ e, por outro, analisar as suas características psicométricas, isto é, verificar a fidelidade - consistência interna - e recolher certos índices de validade, nomeadamente a validade de conteúdo e de construção. Para tal, o IEE foi aplicado à Amostra 2.

\section{Estudo 3: Teste}

Pretendemos nesta fase, por um lado, testar os dados obtidos no Estudo 2, no que concerne à fidelidade e à validade de construção do $I E E$, bem como verifícar 
a sua validade preditiva, relacionando-a com variáveis, como o rendimento e a história escolar. Para tal, o IEE foi administrado à Amostra 3.

\section{RESULTADOS}

\section{Estudo 1}

A análise efectuada às respostas dos sujeitos com apoio no Questionário de Avaliação de Instrumentos levou-nos a proceder a algumas reformulações, em 3 itens, que suscitaram algumas dúvidas em termos de compreensão.

\section{Estudo 2}

A análise estatística dos dados, levada a cabo neste segundo estudo (pré-teste), destinou-se a obter estimativas da qualidade do instrumento, nomeadamente em relação à fidelidade (consistência interna) e à validade de conteúdo e de construção ${ }^{10}$.

Os itens do IEE foram, em primeiro lugar, submetidos a uma análise factorial ", que permitiu evidenciar a estrutura da relação entre eles e identificar as dimensões psicológicas que lhes são subjacentes. Deste modo, a selecção dos itens para a versão final teve em conta os seguintes critérios:

- excluir os itens que não saturem, a l.40l, pelo menos em um dos factores (originados na $1 .^{a}$ análise de componentes principais): solução não rodada;

- excluir os itens que não saturem, a $\mid .335$ |, pelo menos em um dos factores (originados numa posterior análise de componentes principais): solução rodada;

- excluir os itens cuja correlação item-total é inferior a .30;

- excluir os itens que diminuem a consistência interna;

- excluir os itens que apresentem uma correlação $\geq .50 \mathrm{com}$ a Escala de Desejabilidade Social ${ }^{12}$.

\section{Análise factorial}

O procedimento de factorização (método de extracção do factor) utilizado foi o da Análise de Componentes Principais (ACP), seguido de Rotação Ortogonal Varimax (método de transformação).

Uma vez que se trata da adaptação de um instrumento do qual são conhecidas algumas características psicométricas, possuíamos, à partida, uma noção de quantos factores seria possível encontrar. No entanto, decidimos, em primeiro lugar, utilizar como regra para a extracção de factores o critério do valor próprio igual ou superior a 1 (estabelecido por Kaiser, 1965, 1970, in Feldman \& Gagnon, 1986), o que nos permitiu extrair, inicialmente, uma solução com treze factores. Nesta solução obtivemos um MSA (measures of variable sampling adequacy) de .708 que, sendo superior a .50 , indica que os nossos dados (40 itens) represen- 
tam um conjunto de variáveis homogéneas, aspecto este importante para a realização da análise.

Foi obtida uma comunalidade final elevada (65.8), o que significa que os 13 factores explicam $65.8 \%$ da variância total (Tinsley \& Tinsley, 1987), sendo que o $1 .^{\circ}$ factor é o que explica uma maior variância (13.2\%). A partir desta análise foram, então, eliminados os seguintes itens, cuja saturação não atingiu $.40 \mathrm{em}$ nenhum dos 13 factores: $1,2,25,26,34$ e 35 .

Os itens retidos (total de 34) foram submetidos a uma nova ACP com Rotação Ortogonal Varimax, o que resultou numa solução com dez factores. Esta e outras soluções que ensaiámos de seguida, utilizando como critério de extracção de factores o User Specified (critério que implica a explicitação do número de factores), com nove, oito, sete, seis e cinco factores, evidenciaram-se «complexas» (existência de factores apenas com um ou dois itens) e de difícil interpretação. Em consequência, a solução com quatro factores foi escolhida como a melhor representação dos dados. Este resultado encontra-se, aliás, em consonância com a conclusão retirada pelo autor do $I E E$, uma vez que este também extraiu uma solução com apenas quatro factores.

Nesta última análise, foi encontrado um MSA (measures of variable sampling adequacy) de .74. Como este valor ultrapassa largamente .50 , podemos mais uma vez assegurar que os nossos dados representam um conjunto de variáveis homogéneas (Kaiser, 1970, in Feldman \& Gagnon, 1986).

Verificámos que o $1 .^{\circ}$ factor explica $15 \%$ da variância comum dos resultados; o $2 .^{\circ}$ factor, $11.6 \%$; o $3 .^{\circ}$ factor, $7.1 \%$ e o $4 .^{\circ}$ factor, $6.2 \%$, totalizando $39.9 \%$ da variância total. Com efeito, o $1^{\circ}$ factor é essencialmente saturado pelos itens de Integração; o $2 .^{\circ}$ pelos de Coping; o $3^{\circ}$ pelos de Monitorização e o $4 .^{\circ}$ pelos de Repetição (cf. Quadro 1).

Observando de forma mais minuciosa as saturações mais elevadas de cada item com os quatro factores, constata-se que só o item 21 não mantém relações de intensidade razoável com nenhum dos quatro factores ${ }^{13}$. Assim, é pertinente a sua exclusão.

Além disso, determinados itens $(24,30$ e 31$)$ saturam mais intensamente o factor representativo de outra subescala, o que sugere a oportunidade de transferência de itens duma subescala para outra, desde que tais transferências sejam lógicas e conceptualmente aceitáveis. Impõe-se uma análise prévia dos itens em causa.

$\mathrm{O}$ item 24 (Tenho dificuldades em interpretar as perguntas de um teste escrito), apesar de pertencer à subescala Coping, ao ter inerente a tomada de consciência das dificuldades perante situações de avaliação, pode conduzir à identificação de estratégias de «reparação» para ultrapassar essas dificuldades. Assim, a sua transferência para a subescala Monitorização parece-nos justificada, com a respectiva alteração na sua cotação. 
$\mathrm{O}$ item 30 ( Quando não compreendo um texto ou o que se disse na aula, geralmente sei o que fazer para ajudar a minh a compreensão), apesar de ter uma maior saturação no factor 1 , em termos conceptuais consideramos que deve permanecer no factor de origem (Monitorização), já que a sua saturação neste factor é de .364 .

Quadro 1

SATURAÇÃO FACTORIAL MAIS ELEVADA DE CADA ITEM, COMUNALIDADES, VALORES PRÓPRIOS E VARIÂNCIA EXPLICADA, DA SOLUÇÃO OBTIDA COM OS 34 ITENS DO IEE, APÓS ROTAÇÃO VARIMAX,COM EXTRACÇÃO DE COMPONENTES PRINCIPAIS $(\mathrm{N}=225)$

\begin{tabular}{|c|c|c|c|c|c|}
\hline Itens & Factor 1 & Factor 2 & Factor 3 & Factor 4 & $h^{\mathbf{i}^{2}-}$ \\
\hline $\begin{array}{c}3 \\
4 \\
5 \\
6 \\
7 \\
8 \\
9 \\
10 \\
11 \\
12 \\
13 \\
14 \\
15 \\
16 \\
17 \\
18 \\
19 \\
20 \\
21 \\
22 \\
23 \\
24 \\
27 \\
28 \\
29 \\
30 \\
31 \\
32 \\
33 \\
36 \\
37 \\
38 \\
39 \\
40\end{array}$ & $\begin{array}{l}.357 \\
.710 \\
.368 \\
.573 \\
.659 \\
\\
.619 \\
\\
.359 \\
\\
.418 \\
.444 \\
.548 \\
.577 \\
.737 \\
.580\end{array}$ & $\begin{array}{c}.328 \\
. \\
\\
\\
\\
\\
.379 \\
.764 \\
.790 \\
.763 \\
.771\end{array}$ & $\begin{array}{l}.353 \\
. .465 \\
.356 \\
.549 \\
.591 \\
.364 \\
\\
.627 \\
.655\end{array}$ & $\begin{array}{l}.607 \\
.471 \\
.555 \\
.699 \\
.688\end{array}$ & $\begin{array}{l}.391 \\
.552 \\
.258 \\
.346 \\
.489 \\
.417 \\
.403 \\
.447 \\
.417 \\
.286 \\
.556 \\
.324 \\
.490 \\
.437 \\
.415 \\
.411 \\
.620 \\
.374 \\
.186 \\
.171 \\
.219 \\
.284 \\
.166 \\
.401 \\
.367 \\
.353 \\
.242 \\
.410 \\
.445 \\
.190 \\
.588 \\
.632 \\
.615 \\
.643\end{array}$ \\
\hline $\begin{array}{c}\text { VALORES } \\
\text { PRÓPRIOS } \\
\text { \% VAR. } \\
\text { TOTAL }\end{array}$ & $\begin{array}{l}5.087 \\
15\end{array}$ & $\begin{array}{c}3.937 \\
11.6\end{array}$ & $\begin{array}{l}2.42 \\
7.1\end{array}$ & $\begin{array}{l}2.102 \\
6.2\end{array}$ & $\begin{array}{l}13.546 \\
39.9\end{array}$ \\
\hline
\end{tabular}


Em relação ao item 31 (Tenho problemas em me automotivar para iniciar o estudo), consideramos adequada a sua permanência no factor de origem (Coping), apesar de ter saturação mais saliente no factor Repetição (-.370) do que no factor Coping (.297).

Após estas transformações, o IEE ficou com 33 itens: a subescala Integração foi alargada para 13 itens, as subescalas Repetição e Coping foram reduzidas para 6 itens, e a subescala Monitorização foi também reduzida para 8 itens.

Assim, podemos afirmar que, por um lado, os resultados da análise factorial permitem concluir que as quatro subescalas cobrem conjuntos de características relativamente homogéneas e, por outro, que a estrutura factorial confirma o modelo teórico subjacente.

Tais resultados constituem, também, uma garantia da validade de conteúdo e de construção do instrumento.

\section{Análise psicométrica}

Os itens do $I E E$, retidos através da análise factorial, foram submetidos à análise psicométrica (coeficiente alpha de Cronbach) que incidiu, não sobre o instrumento na sua globalidade, mas nas suas quatro subescalas. A análise da fidelidade revela, em termos gerais, uma consistência interna razoável (de .635 a .803), o que se traduz numa boa homogeneidade dos itens nas diferentes subescalas.

\section{Estudo 3}

Este estudo teve os seguintes objectivos: (1) reiterar a estimativa da qualidade do IEE obtida no Estudo 2; (2) avaliar a validade preditiva e concorrente do IEE; e (3) verificar a relação entre as variáveis consideradas nesta investigação.

No sentido de concretizar o primeiro objectivo, procedemos novamente à análise factorial do IEE. Apesar de ser apenas de cariz confirmatório, fizemos correr várias análises (algumas não passíveis de interpretação) que nos permitiram encontrar dez, oito, seis, cinco e quatro factores ${ }^{14}$. Esta última solução (quatro factores) foi mais uma vez escolhida como a melhor representação dos dados, $o$ que, aliás, vai de encontro aos resultados obtidos no Estudo 2.

Tendo em conta o conteúdo dos itens que saturam nos diferentes factores, constatámos que, tal como na análise efectuada no Estudo 2, a configuração factorial é idêntica à encontrada em estudos anteriores (v.g., Moreno \& Di Vesta, 1991).

\section{Análises descritiva e inferencial}

\section{Análise descritiva}

\section{a) Rendimento escolar}

Considerámos a média aritmética das classificações obtidas pelos estudantes, nos anos precedentes, como indicador do rendimento escolar. A análise descritiva revela que as médias se situam entre $10(1.2 \%)$ e $15(7.2 \%)$ valores, sendo a 
mais frequente de $13(33.2 \%)$, seguida da de $12(29.3 \%)$, da de $11(16.5 \%)$ e da de $14(12.6 \%)$.

\section{b) História escolar}

Considerámos o número de reprovações até ao momento como indicador da história escolar ${ }^{15}$. A análise descritiva, através do cálculo de percentagens, revelou que a maioria dos alunos transitou sempre de ano $(47.59 \%), 31.51 \%$ reprovou uma vez, $9.97 \%$ reprovou 2 vezes e $10.93 \%$ reprovou três vezes.

c) Estratégias de estudo

Pelo facto de as subescalas do $I E E$ não possuírem um número equitativo de itens, procedemos, também, ao cálculo da média e desvio-padrão ajustados, de modo a ter uma ideia mais realista dos resultados (cf. Quadros 2 e 3 ) $^{16}$.

\section{Quadro 2}

MÉDIAS, DESVIOS-PADRÃO, MÍNIMO, MÁXIMO, MÍNIMO-MÁXIMO POTENCIAIS PARA A TOTALIDADE DA AMOSTRA $(\mathrm{N}=311)$

\begin{tabular}{l|ccccc}
\hline Subescalas & Média & DP & Mín. & Máx. & $\begin{array}{c}\text { Min-Máx } \\
\text { Potenciais }\end{array}$ \\
\cline { 1 - 5 } Integração & 44.203 & 7.163 & 20 & 59 & $13-65$ \\
Repetição & 20.672 & 4.148 & 8 & 30 & $6-30$ \\
Monitorização & 29.154 & 3.002 & 20 & 38 & $8-40$ \\
Coping & 20.466 & 4.049 & 11 & 29 & $6-30$ \\
\hline
\end{tabular}

Quadro 3

MÉDIAS, DESVIOS-PADRÃO AJUSTADOS, MÍNIMO, MÁXIMO, PARA A TOTALIDADE DA AMOSTRA $(\mathrm{N}=311)$

\begin{tabular}{l|llcc}
\hline Subescalas & Média & DP & Mínimo & Máximo* \\
\cline { 1 - 4 } Integração & 1.95 & .243 & 1.176 & 2.524 \\
Repetição & 1.981 & .319 & 1.085 & 3.142 \\
Monitorização & 2.054 & .174 & 1.571 & 2.691 \\
Coping & 1.964 & .309 & 1.301 & 2.774 \\
\hline
\end{tabular}

* Nota: Com o ajustamento das médias, o mínimo e máximo potencial é igual para todas.

Tal procedimento permitiu verificar que a subescala mais pontuada foi a Monitorização, seguida da Repetição, da Coping e, por fim, da Integração. Numa primeira conclusão, podemos realçar que, apesar dos alunos manifestarem utilizar 
em maior número as estratégias de monitorização, há uma maior tendência para empreenderem estratégias de repetição e menos de integração.

\section{Análise inferencial}

Recorremos aos seguintes procedimentos: inicialmente, calculámos as correlações entre os possíveis preditores (estratégias de estudo) e o critério (média escolar e história escolar) ${ }^{17} \mathrm{e}$, posteriormente, realizámos análises de regressão.

O cálculo das correlações que o rendimento escolar estabelece com as variáveis consideradas permitiu chegar aos seguintes resultados: está correlacionado positiva e significativamente com as subescalas Integração e Monitorização $(\mathrm{r}=.394, p<.001$ e $\mathrm{r}=.375, p<.001$, respectivamente); está correlacionado negativa e significativamente com a subescala Coping $(\mathrm{r}=-.313, p<.001)$; está correlacionado negativa, mas não significativamente, com a subescala Repetição $(\mathrm{r}=.096)$.

O cálculo da regressão múltipla das quatro variáveis em relação ao rendimento escolar permitiu verificar que estas variáveis explicam $27.6 \%$ da variância do rendimento escolar $\left(r=.525 ; r^{2}=.276 ; p<.001\right)$.

A variável Integração representa o preditor mais poderoso do rendimento escolar, pois explica por si só $15.5 \%$ da variância. A variável Coping explica $7 \%$, a variável Monitorização $4.3 \%$ e a variável Repetição explica .8\%.

De modo a facilitar a visualização dos resultados, apresentamos as Rectas de Regressão.

\section{Gráfico 1}

RECTAS DE REGRESSÃO DAS CORRELAÇÕES ENTRE AS SUBESCALAS DO IEE E A MÉDIA ESCOLAR
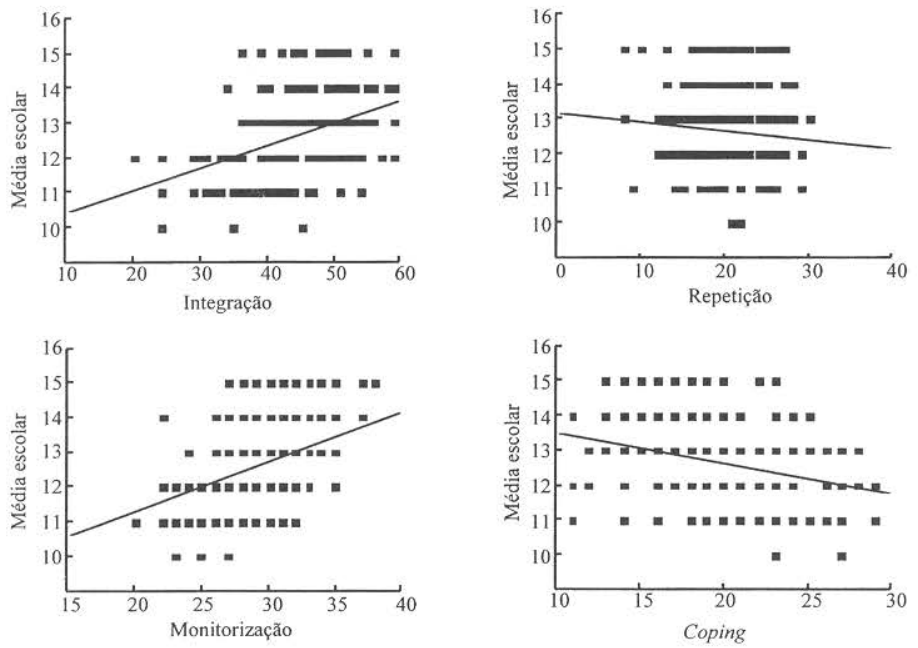
A observação das rectas de regressão leva-nos a concluir que há um aumento proporcional da utilização de estratégias de Integração e de Monitorização, relativamente à média escolar, ocorrendo o inverso em relação às estratégias Repetição e Coping.

O cálculo das correlações, que a história escolar estabelece com as variáveis consideradas, permitiu chegar aos seguintes resultados: está correlacionada positiva e significativamente com a subescala Repetição $(r=.133, p<.05)$; está correlacionada negativa, mas não significativamente, com as subescalas Integração e Monitorização $(\mathrm{r}=-.028 ; \mathrm{r}=.095$, respectivamente); está correlacionada positiva, mas não significativamente, com a subescala Coping $(r=.008)$.

Estes resultados levam-nos a concluir que os alunos que referem utilizar mais estratégias de Repetição são os que têm maior número de reprovações durante o percurso escolar. As outras correlações observadas podem ser consideradas desprezíveis $^{18}$.

Os resultados obtidos evidenciam uma influência recíproca entre o rendimento escolar e estratégias de estudo. Mais especificamente, são os alunos com rendimento superior que manifestam a utilização de estratégias de integração e a monitorização do estudo.

Apesar de ter sido encontrada uma correlação negativa entre rendimento escolar e a manifesta utilização da estratégia de repetição, esta não é significativa. Por outro lado, o rendimento escolar encontra-se negativa e significativamente correlacionado com a dificuldade em lidar com situações ansiógenas.

\section{DISCUSSÃO}

Um dos principais objectivos da presente investigação foi a adaptação, a nível do ensino universitário, de um instrumento capaz de medir, de modo fiel e válido, quatro conjuntos de estratégias de estudo, representativos dos processos de controlo do sistema de processamento de informação - o Cognitive Skills Inventory.

A partir da análise psicométrica efectuada, podemos afirmar que as 4 subescalas que constituem o Inventário de Estratégias de Estudo (Integração, Repetição, Monitorização e Coping) possuem uma fidelidade adequada, em termos de homogeneidade dos itens. A análise factorial apoiou, não só a consistência interna, mas também a validade discriminante das diferentes subescalas.

As análises da validade de conteúdo e de construção evidenciaram resultados que nos permitem afirmar que o IEE avalia com rigor as estratégias de estudo. A validade preditiva foi, também, assegurada, apesar de não ter sido encontrada uma correlação significativa entre a subescala Repetição e o Rendimento Escolar. Foi observada, no entanto, a existência de uma correlação positiva e significativa entre a manifesta utilização deste tipo de estratégia e a História Escolar. Realça- 
mos, assim, a utilidade da consideração deste instrumento para a compreensão das variações do rendimento escolar no ensino universitário.

Por último, é de referir que a validade externa dos resultados obtidos é limitada aos estudantes da Faculdade de Letras - Ramo de Formação Educacional da Universidade de Coimbra.

No que concerne aos restantes resultados encontrados, podemos verificar que os alunos com melhores resultados escolares referem utilizar, com mais frequência, estratégias de Integração (que apelam a um "processamento profundo", segundo Di Vesta) e manifestam uma maior monitorização do estudo assim como um nível de ansiedade mais baixo. Por outro lado, os alunos que possuem um número mais elevado de reprovações durante o percurso escolar referem utilizar, com mais frequência, estratégias de Repetição.

A associação de tendência positiva encontrada entre a utilização de estratégias de Monitorização e o rendimento escolar realça que são os melhores alunos que detêm a faculdade de planificar, de dirigir a compreensão e de avaliar o que foi aprendido, isto é, são aqueles que mais utilizam as ditas estratégias metacognitivas, avaliadas no IEE pela subescala Monitorização. Este resultado é compreensível neste nível de escolaridade.

Esta verificação vai de encontro à constatação, já efectuada por outros autores, da existência de diferenças significativas no desempenho escolar, não apenas em função da utilização de estratégias cognitivas, mas também de estratégias metacognitivas (v.g., Weinstein, 1994), o que os levou a concluir que os bons alunos são mais aptos tanto na utilização de estratégias para adquirir, otganizar e utilizar o seu conhecimento, como na regulação do próprio progresso cognitivo.

Verificámos, também, que o rendimento escolar se apresenta negativamente relacionado com a ansiedade manifesta, avaliada no IEE pela subescala Coping.

Atendendo às limitações inerentes ao carácter do nosso estudo (transversal e correlacional), consideramos que os resultados obtidos devem ser interpretados de forma cautelosa. De facto, o recurso a um plano deste tipo, para além de não permitir conhecer a direç̧ão das relações observadas, implica, também, um controlo menos rigoroso das variáveis estranhas, com o consequente aumento de probabilidade das ameaças à validade interna (rigor do plano) e externa (possibilidade de generalização dos resultados).

\section{CONCLUSÃO}

Apesar das limitações referidas e da necessidade de considerar outros aspectos em futuras investigações, os resultados obtidos permitiram observar que a utilização de determinadas estratégias de estudo constitui um bom preditor do sucesso escolar. Salienta-se, assim, a necessidade de uma incidência educativa 
mais atenta, neste aspecto, não apenas no ensino superior, mas também em níveis de escolaridade mais baixos.

Para além desta implicação de ordem geral, são de salientar outras implicações de carácter mais particular:

Diversos estudos têm demonstrado que o ensino de estratégias origina um aumento do desempenho escolar (v.g., Dansereau, 1985; Palincsar \& Brown, 1984; Weinstein \& Mayer, 1986) e que pode funcionar tanto como um meio de intervenção «remediativa» como «preventiva» (McKeachie, 1988). Pensamos, assim, ser importante incrementar, nos diferentes níveis de ensino, estratégias do foro cognitivo e metacognitivo e do foro afectivo/motivacional. Esta tarefa deverá ser desenvolvida numa estreita colaboração entre psicólogos e professores.

Tal como já acontece em algumas escolas do nosso país, ao nível do $3 .^{\circ}$ ciclo do ensino básico, consideramos de grande interesse fomentar programas de treino de estratégias que, em nosso entender, beneficiarão ser empreendidos em contexto de sala de aula, nomeadamente com alunos de níveis de escolaridade mais baixos.

Ao nível do ensino universitário e à semelhança de algumas experiências realizadas noutros países (v.g., EUA), parece-nos útil a implementação de um serviço de aconselhamento nas Universidades, a funcionar fundamentalmente junto dos estudantes do primeiro ano, uma vez que serão estes que, em princípio, mais necessitam de orientação na utilização de estratégias de processamento de informação, na planificação do estudo, na monitorização da aprendizagem, no controlo do nível de ansiedade, etc. Por outro lado, os Guias de Estudantes, também à semelhança do que acontece noutros países (v.g. Bélgica), poderão ter um papel importante nesta intervenção se contiverem, para além das informações mais comuns (por exemplo, objectivos, conteúdos programáticos, modalidades de avaliação, referências bibliográficas de cada disciplina), orientações mais direccionadas para o estudo estratégico (por exemplo, como ter acesso à(s) biblioteca(s), planificar o tempo de estudo, realizar um trabalho escrito, organizar a época de exames e, mesmo algumas estratégias de processamento da informação: tirar apontamentos, fazer sumários, esquemas, etc.).

Encorajar o aluno a ser responsável pela sua própria aprendizagem, desenvolvendo o que tem sido denominado de «consciência metacognitiva» (Entwistle, 1990a, 1990b), parece ser um aspecto importante em termos de realização escolar. Este resultado a que chegámos não minimiza o papel do professor. Pelo contrário, este deve assumir a orientação dessa responsabilidade, cabendo-lhe acompanhar o processo de aprendizagem, nomeadamente através da observação cuidada dos modos de conceptualização das informações trabalhadas, da sugestão de certas estratégias de estudo, no encorajamento da autonomia, etc.. Para além deste acompanhamento e não descurando a importância do conhecimento dos conteúdos programáticos e os métodos/processos de transmissão da informação, o professor deve, também, definir claramente os objectivos a atingir pelos alunos, 
as modalidades de avaliação, de modo que estes possam adaptar e regular os próprios processos de aprendizagem.

Deste modo, e tal como já foi referido, os objectivos da Educação não devem incidir apenas na aquisição de conhecimentos, mas também no desenvolvimento de processos e mecanismos de aprendizagem que possibilitem ao aluno a construção activa dos seus próprios conhecimentos. Como referiu Nicolau Raposo $\left({ }^{2} 1995,254\right)$, é necessário que os estudantes "interv[enham] mais activamente no processo da sua aprendizagem" e que, relativamente a esta, "assumam mais directamente as [suas] responsabilidades". Só esta mudança estrutural na organização e desenvolvimento curriculares poderá promover a aprendizagem activa e responsável e, correlativamente, aumentar o sucesso escolar.

\section{NOTAS}

* Este artigo é parte integrante da Dissertação de Mestrado em Psicologia (área de especialização em Psicologia Pedagógica), apresentada à Faculdade de Psicologia e de Ciências da Educação da Universidade de Coimbra, a qual foi elaborada sob a orientação do Prof. Doutor Nicolau Vasconcelos Raposo, a quem agradecemos a supervisão científica que nos dispensou.

1 Sobre as perspectivas behaviorista e cognitivista do processo de aprendizagem, ver Ribeiro (1997).

2 Este autor é um dos responsáveis pela abordagem cognitiva da memória ao atribuir ao sujeito um papel activo na construção da informação e experiência.

3 Paris (1988) realça que a popularidade de estratégias de estudo como o SQ3R (Robinson, 1946) e os estudos pioneiros no «hypothesis testing» em crianças (v.g., Bruner et al., 1956) serviram também para fomentar as investigações que surgiram posteriormente neste âmbito: estratégias de organização mnemónicas (Tulving, 1962), codificação visual [de imagens] (Paivio, 1970), aprender a aprender (Postman, 1969), mnemónicas e estratégias visuais [imagens] (Flavell, 1970), autocontrolo comportamental (Thoreson \& Mahoney, 1974), aprendizagem generativa (Wittrock, 1974), elaboração (Rohwer, 1970), entre outras. Igualmente, os psicólogos desenvolvimentistas, influenciados especialmente por Flavell - psicólogo cognitivo-desenvolvimentista - se interessam pela utilização de estratégias, definidas como actividades deliberadas ao serviço da aprendizagem e do desenvolvimento.

4 Sobre as terminologias e taxonomias, ver Ribeiro (1994).

5 Por exemplo, na preparação para uma situação de avaliação, um aprendiz pode usar autoverbalizações positivas para reduzir sentimentos de ansiedade; na aprendizagem de pares associados, pode formar uma imagem mental para ajudar a associação dos objectos representados; na aprendizagem de uma passagem de um texto, pode elaborar um sumário de cada secção; na 
aprendizagem de um conceito científico, pode tirar notas sobre as informações mais importantes. Estas actividades constituem exemplos de estratégias.

6 Sendo que no Estudo 2 (pré-teste) foram seleccionados 225 estudantes: 171 do sexo feminino $(76 \%)$ e 54 do sexo masculino (24\%). As suas idades oscilavam entre os 20 e os 30 anos, situando-se a maior percentagem na faixa etária dos $22-23$ anos $(40.44 \%)$, seguida da faixa etária dos $20-21$ anos $(34.67 \%)$. Relativamente ao ano de frequência, verifica-se um equilíbrio na distribuição dos sujeitos, ou seja, $118(52.44 \%)$ frequentavam o $4^{\circ}$ ano e $107(47.56 \%)$ o $3 .^{\circ}$ ano.

No Estudo 3 (teste) foram seleccionados 311 estudantes: 219 do sexo feminino (70.42\%) e 92 do sexo masculino (29.58\%). As suas idades oscilavam entre os 20 e os 26 anos, a maioria (47.58\%) situava-se na faixa etária dos $22-23$ anos, seguida da faixa etária dos $20-21$ anos $(21.87 \%)$. Desses estudantes, $46.30 \%$ frequentava o $3 .^{\circ}$ ano e $58.70 \%$ frequentava o $4 .^{\circ}$ ano.

7 Estes itens, que estão directamente relacionados com a metacognição, reportam-se: (1) ao estabelecimento de objectivos ou metas de estudo, (2) à monitorização da escolha de estratégias de acordo com os objectivos estabelecidos; (3) à avaliação da compreensão resultante; e (4) à alteração de estratégias. Sobre metacognição, ver Ribeiro (1997).

8 Como foi referido anteriormente, o processamento profundo de informação, conceito proposto por Craik \& Lockhart (1972), origina traços de memória mais duradoiros do que o processamento superficial de informação. Estes autores supõem que os traços de memória resultam de actividades de processamento e variam ao longo de um contínuo: do superficial (no qual o estímulo físico é o único objecto de atenção) ao profundo (no qual os significados e as associações conceptuais são processados).

9 A definição de coping (de origem anglo-saxónica) não é tarefa fácil. A tradução literal para o português remete para expressões como: «formas de lidar com», ou «estratégias de confronto». Sendo, porém, tal termo bastante usado em diversos domínios (designadanente o da terapia comportamental), optámos, à semelhança de outros autores (v.g., Pereira, 1991), pelo uso do termo no original.

10 Procedemos apenas a uma aplicação da primeira versão, já que seria praticamente impossível voltar a contactar os mesmos sujeitos. Contudo, a consistência temporal das escalas, regra geral, testada a partir da estratégia teste-reteste, pôde ser assegurada pelo facto de utilizarmos como índice de fidelidade - índice de consistência interna - o coeficiente alpha de Cronbach, considerado a estimativa por defeito, estimativa mais baixa do coeficiente teste-reteste (Nunnally, 1978).

"A análise factorial surge neste estudo com o propósito de avaliar o grau de validade factorial da adaptação portuguesa do Inventário de Estratégias de Estudo, ou seja, verificar se os itens de cada subescala cobrem um conjunto de características homogéneas (consistência interna), e se as quatro subescalas avaliam dimensões psicológicas distintas (validade discriminante).

12 No sentido de controlar o efeito da desejabilidade social e proporcionar mais uma possibilidade de verificação da validade de construção, procedeu-se à análise da correlação entre cada item do IEE e a Escala de Desejabilidade Social de Crowne \& Marlowe (1960), bem como entre este e as diferentes subescalas que o constituem. As correlações encontradas foram, na generalidade, baixas. De salientar que a correlação mais elevada entre os itens do IEE e o EDS foi de $-.196, p<.01$ e entre as subescalas do $I E E$ e o $E D S$ é de -.101 . 
13 A não existência de critérios universais, para determinar o nível de significância dos coeficientes de saturação exigidos para a manutenção das variáveis na escala identificada pelo factor, levou-nos a optar pela opinião de Stevens (1986) que considera um valor crítico na determinação das saturações significativas, o qual tem em conta o tamanho da amostra. Desta forma, para uma amostra de 225 sujeitos, a um nível de significância de .01, este valor crítico é de l.173|. Este autor refere, ainda, que o valor crítico deve ser multiplicado por 2 para testar a significância da saturação. Assim, consideraremos como significativo um coeficiente de saturação $\geq 1.335$.

14 Nesta análise utilizamos como critério de extracção dos factores o User Specified - critério que implica a explicitação do número de factores a serem obtidos.

15 Consideraram-se apenas os insucessos que impediam a passagem de ano, excluindo-se as reprovações em disciplinas específicas.

16 O ajustamento das médias foi realizado através do cálculo da proporção entre a pontuação total real e o número de itens de cada subescala. Para tal, foi utilizada uma fórmula proposta por Howell (1987, p. 304): $Y=2 \operatorname{arcsine} \sqrt{\boldsymbol{p}}$.

17 De acordo com Nunnaly (1978), o preditor só é válido quando é encontrada uma correlação significativa com o critério. No nosso caso, devemos referir que a escolha do preditor e do critério teve em conta o nosso interesse em saber em que medida a variabilidade nas subescalas do $I E E$ contribui para a variabilidade na média e na história escolar.

18 Não procedemos à análise de regressão, visto que, de entre as variáveis consideradas, apenas uma apresentou correlação significativa com a história escolar.

\section{REFERÊNCIAS BIBLIOGRÁFICAS}

BENET, R., ANDRADA, B., ALVAREZ, J., \& BELLON, F. (1990), Eficácia no estudo, Tradução de Rui Paulo, Rio Tinto, Edições Asa.

BIGGS, J. B. (1984), "Learning strategies, student motivation patterns, and subjectively perceived success", in J. R. Kirby (Ed.), Cognitive strategies and educational performance, Orlando, Fl. Academic Press.

BROOKS, L. W., SIMUTS, Z., \& ONNEIL, Jr. (1985), "The role of individual differences in learning strategies research", in R. Dillon (Ed.), Individual differences in cognition (Vol. 2), New York, Academic Press.

CAMP, W. G. (1990), "Participation in student activities and achievement: A covariance structural analysis", Journal of Educational Research, 83 (5), 272-278.

CARVALHO, R., MOREIRA, L., SANCHES, J., \& SANTOS, M. T. (1987), "Desenvolvimento de competências de estudo nos jovens". Cadernos de Consulta Psicológica, 3, 89-94.

CRAIK, F. I. M., \& LOCKHART, R. S. (1972), "Levels of processing: a framework for memory research", Journal of Verbal Learning and Verbal Behavior, 11, 671-684. 
CROWNE, D. P., \& MARLOWE, D. (1960), “A new scale of social desirability independente of psychopathology", Journal of Consulting Psychology, 24 (4), 349-354.

DANSEREAU, D. F. (1978), "The development of a learning strategies curriculum", in H. F. OíNeil, Jr. (Ed.), Learning strategies. New York, Academic Press.

DANSEREAU, D. F. (1985), "Learning strategy research”, in J. W. Segal, S. F. Chipman, \& R. Glaser (Eds.), Thinking and learning skills (Vol. 1). Hillsdale, NJ, Erlbaum.

DANSEREAU, D. F. (1988), "Cooperative learning strategies”, in C. E. Weinstein, E. T. Goetz, \& P. A. Alexander (Eds.), Learning and study strategies: Issues in assessment, instruction and evaluation. New York, Academic Press.

DI VESTA (1986), The cognitive skills inventory (in press).

ELSHOUT, J., \& VEENMAN, M. (1992), "Relation between intellectual ability and working method as predictors of learning", Journal of Educational Research, 85 (3), 134-143.

ENTWISTLE, N. (1990a), "Introduction: changing conceptions of learning and teaching", in N. Entwistle (Ed.), Handbook of educational ideas and practices, London, Routledge.

ENTWISTLE, N. (1990b), “Teaching and the quality of learning in higher education", in N. Entwistle (Ed.), Handbook of educational ideas and practices, London, Routledge.

FELDMAN, D., \& GAGNON, J. (1986), Statview 512+TM, Calabasas, Abacus Concepts Inc.

FONTAINE, A. M. (1990), “Teste de Motivação para a Realização de J. M. H. Hermans: Adaptação para jovens portugueses", Psychologica, 4, 81-103.

HOWELL, D. C. (1987), Statistical methods for psychology, Boston, PWS-KENT Publishing Company.

LENS, B. K. (1992), "Self-managed learning strategy systems for children and youth", School Psychology Review, 21 (2), 211-228.

LEVIN, J. R., \& PRESSLEY, M. (1985), "Mnemonic vocabulay instruction: Whatís fact, whatís fiction", in R. F. Dillon (Ed.), Individual differences in cognition (Vol. 2). New York: Academic Press.

MAYER, R. E. (1988), "Learning strategies: An overview", in C. E. Weinstein, E. T. Goetz, \& P. A. Alexander (Eds.), Learning and study strategies: Issues in assessment, instruction, and evaluation. New York, Academic Press.

McKEACHIE, W. J. (1988), "The need for study strategy training", in C. E. Weinstein, E. T. Goetz, \& P. A. Alexander (Eds.), Learning and study strategies: Issues in assessment, instruction, and evaluation, New York, Academic Press.

MORENO, V., \& DI VESTA, F. J. (1991), “Cross-cultural comparisons of study habits”. Journal of Educational Psychology, 83 (2), 231-239.

NUNNALLY, J. (1978), Psychometric theory (2nd ed.), New York, McGraw-Hill.

PALINCSAR, A. S., \& BROWN, A. L. (1984), "Reciprocal teaching of comprehension-fostering and monitoring activities", Cognition and Instruction, 1, 117-175.

PARIS, S. G. (1988), “Models and metaphors of learning strategies", in C. E. Weinstein, E. T. Goetz, \& P. A. Alexander (Eds.), Learning and study strategies: Issues in assessment, instruction, and evaluation, New York, Academic Press. 
PARIS, S. G., LIPSON, M. Y., \& WIXSON, K. K. (1983), "Becoming a strategic reader", Contemporary Educational Psychology, 8, 293-316.

PARIS, S., \& WINOGRAD, P. (1990), "How metacognition can promote academic learning and instruction", in B. Jones \& L. Idol (Eds.), Dimensions of thinking and cognitive instruction, New Jersey, Erlbaum.

PEREIRA, A. (1991), Coping, auto-conceito e ansiedade social (sua relação com o rendimento escolar). (Dissertação de Mestrado apresentada à Faculdade de Psicologia e de Ciências da Educação da Universidade de Coimbra.

PINTRICH, P. R., \& DE GROOT, E. V. (1990), "Motivational and self-regulated learning components of classroom academic performance", Journal of Educational Psychology, 82 (1), 33-40.

RAPOSO, N. V. ( $\left.{ }^{2} 1995\right)$, Estudos de psicopedagogia, Coimbra, Coimbra Editora.

RIBEIRO, C. (1994), Contributo para o esclarecimento de processos intervenientes na aprendizagem: Estratégias de estudo e locus de controlo (Dissertação de Mestrado apresentada à Faculdade de Psicologia e de Ciências da Educação da Universidade de Coimbra, minreog.).

RIBEIRO, C. (1997), "Estudo do processo de aprendizagem: Duas perspectivas", Revista Máthesis, $\mathrm{n}^{\circ} 6$.

SILVA, A. L., \& SÁ, I. (1989), “Um programa para o desenvolvimento de estratégias de estudo. Reflexões sobre uma prática clínica”, Revista Portuguesa de Psicologia, 25, 93-108.

SILVA, A. L., \& SÁ, I. (1993), Saber estudar e estudar para saber, Porto, Porto Editora.

SIMPSON, L. M., OLEJNIK, S., YU-WEN Tam, A., \& SUPATTATHUM, S. (1994), "Elaborative verbal rehearsals and college studentsí cognitive performance", Journal of Educational Psychology, 86 (2), 267-278.

STEVENS, J. (1986), Applied multivariate statistics for the social sciences, New Jersey, Erlbaum.

TINSLEY, H. E., \& TINSLEY, D. J. (1987), “Uses of factor analysis in counseling psychology research". Journal of Counseling Psychology, 34 (4), 414-424.

WEINSTEIN, C. E. (1994), "Students at-risk for academic failure: learning to learn classes", in K. Prichard \& R. McLavan Sawyer (Eds.), Handbook of college teaching: Theory and applications (in press).

WEINSTEIN, C. E., \& MAYER, R. E. (1986), "The teaching of learning strategies", in M. C. Wittrock (Ed.), Handbook of research on teaching, New York, Macmillan.

WITTROCK, M. C. (1988), "A constructive review of research on learning estrategies", in C. E. Weinstein, E. T. Goetz, \& P. A. Alexander (Eds.), Learning and study strategies: Issues in assessment, instruction, and evaluation, New York, Academic Press. 